\title{
Performance of an Optimally Spaced PPM Ultra-Wideband System with Direct Sequence Spreading for Multiple Access
}

\author{
Vinod Venkatesan*, Huaping Liu*, Curt Nilsen ${ }^{\dagger}$, Ron Kyker ${ }^{\dagger}$ and Mario E. Magaña* \\ ${ }^{*}$ School of EECS, Oregon State University, Corvallis, OR 97331 USA \\ Email: hliu@ece.orst.edu, Telephone: +1 5417372973 \\ $\dagger$ Instrumentation Systems Engineering Department, Sandia National Labs \\ MS 9101, PO Box 969, Livermore, CA 94550 USA
}

\begin{abstract}
In this paper, we analyze the error performance of an ultra-wideband (UWB) system with pulse position modulation (PPM) for data modulation and direct sequence (DS) spreading for multiple access in indoor lognormal fading channels. A RAKE receiver is used to combine a subset of the resolvable multipath components using the maximal ratio combining technique. Inter-path and multiple-access interferences are modeled and incorporated into the BER expressions. As two special cases, performance of the optimally-spaced and orthogonal signaling schemes are compared. The results presented in this paper allow comprehensive evaluation of system performances with any combination of parameters.
\end{abstract}

\section{INTRODUCTION}

Ultra-wideband (UWB) techniques are attractive for highrate, low-power communications over short distances. UWB systems use extremely narrow pulses to transmit information. The ultra-wide bandwidth makes the channel highly frequency selective, resulting in a large number of resolvable multipath components. The received power is distributed over all these paths, which make diversity reception techniques a necessity for a reliable communication. Multiple access communication systems employing UWB technologies have drawn great commercial and academic interest. Various multiple access schemes and their performances have been reported in literature [1], [2]. Time hopping (TH) has been found to be a good technique for multiple access [1]. Direct sequence (DS) spreading can be applied to UWB systems for multiple access and the performance of a DS UWB system with pulse amplitude modulation (PAM) was analyzed in [2]. Since ultrawideband systems are inherently spread spectrum systems, the use of spreading codes is solely for accommodating multiple users.

Direct sequence can also be used for multiple access in a PPM UWB system. In such a system, each symbol is represented by a series of pulses that are pulse-amplitudemodulated by a chip sequence. Input data are modulated onto the relative positions of each sequence of pulses. Since a series of pulses are used to represent one symbol, multipath components corresponding to a particular symbol have different time shifts and overlap with one another, causing inter-path interference. Thus, each path at the input of the combiner is corrupted by preceding and following paths, which eventually limits the performance. Another major factor governing the performance of this system, like any other PPM-based system, is the set of time-shifts used to represent different symbols. The most commonly used PPM scheme is the orthogonal signaling scheme for which the UWB pulse shape is orthogonal to its time-shifted version. There also exists a set of optimal time shifts for any $M$-ary PPM scheme. In this paper we consider the simplest case of binary PPM signaling for UWB systems with direct sequence spreading for multiple access and compare the performance of the optimally-spaced and the orthogonal signaling schemes in indoor lognormal fading channels.

This paper is organized as follows. Section II describes the transmitter, the channel, and the receiver models. Error performance analysis based on a semi-analytical approach are described in Section III. Numerical results are obtained and discussed in Section IV, followed by concluding remarks in Section V.

\section{SySTEM MODEL}

\section{A. Transmitter Model}

For binary DS PPM systems, information bit 1 is represented by a frame of pulses without any delay and information bit 0 is represented by the same frame of pulses but with a delay $\tau$ relative to the time reference. Let us assume that there are $N_{u}$ users in the system and the user with index $u=1$ is the desired user. The transmitted UWB signal of the $u^{\text {th }}$ user is represented by

$$
s_{u}(t)=\sqrt{\mathcal{E}_{s}} \sum_{i=-\infty}^{\infty} \sum_{n=0}^{N_{c}-1} a_{u, n} w\left\{t-i T_{r}-n T_{c}-\frac{\tau}{2}\left(1-b_{u, i}\right)\right\}
$$

where $\mathcal{E}_{s}$ is the symbol energy, $w(t)$ is the UWB pulse (e.g., a windowed Gaussian monocycle) assumed to have a nonzero value only for a time period $T_{p}, a_{u, n} \in\{ \pm 1\}$ is the $n^{\text {th }}$ chip of the $u^{t h}$ user, $T_{r}$ is the symbol repetition period, $T_{c}$ is the chip duration, $N_{c}$ is the number of chips used to represent one symbol, $b_{u, i} \in\{ \pm 1\}$ is the $i^{\text {th }}$ nonreturn-to-zero converted information bit of the $u^{\text {th }}$ user, and $\tau$ is the time-shift used in PPM. It is also assumed that $T_{c}=\epsilon T_{p}$, where $\epsilon$ is a positive integer. Except the spreading code, which is unique for each 
user, and users' information bits, all other parameters defined above are the same for all users.

To ensure that the inter-symbol interference is negligible, the guard interval, $T_{r}-N_{c} T_{c}$, between adjacent symbols must be large enough for the range of delay spread values of interest. Let the sequence of pulses used to represent a symbol of the $u^{t h}$ user be

$$
\begin{aligned}
& S_{u, 0}(t)=\sum_{n=0}^{N_{c}-1} a_{u, n} w\left\{t-n T_{c}\right\} \\
& S_{u, 1}(t)=S_{u, 0}(t-\tau)
\end{aligned}
$$

where $S_{u, 0}(t)$ and $S_{u, 1}(t)$ have unit energy, i.e., $\int_{-\infty}^{\infty} S_{u, j}^{2}(t) d t=1, j=0,1$. For the orthogonal signaling scheme, $\tau$ is chosen such that $\int_{-\infty}^{\infty} S_{u, 0}(t) S_{u, 0}(t-\tau) d t=0$. In the case of direct sequence spread symbols, the orthogonality condition is met for infinite choices of $\tau$ and the minimum of those values is chosen as the time shift. For optimally-spaced signaling, the time shift $\tau$ is chosen in the range $0 \leq \tau \leq T_{p}$ such that the cross-correlation between the symbols is minimized. Thus, $\tau$ is determined as

$$
\tau \triangleq \underbrace{\arg \min }_{\tau\left(0 \leq \tau \leq T_{p}\right)} \int_{-\infty}^{\infty} S_{u, 0}(t) S_{u, 0}(t-\tau) d t .
$$

\section{B. Channel Model}

The frequency selective UWB channel is modeled as a discrete-time, linear filter with an impulse response [2]

$$
h(t)=\sum_{l=0}^{L-1} \alpha_{l} \delta\left(t-l T_{p}\right)
$$

where $L$ is the total number of resolvable multipath components, $\delta(t)$ is the Dirac delta function, and $\alpha_{l}=\lambda_{l} \beta_{l}$ is the fading coefficient corresponding to the $l^{\text {th }}$ multipath. The parameter $\lambda_{l} \in\{ \pm 1\}$ with equal probability accounts for the random pulse inversion that can occur due to reflections [3]. For indoor channels, the magnitude term $\beta_{l}$ is lognormally distributed [5]. The minimum multipath resolution in this model is equal to the pulse width $T_{p}$. It is assumed that there is a resolvable multipath component every $T_{p}$.

\section{Receiver Model}

After passing through the channel, each user's signal arrives at the receiver as multiple independently faded copies. The received signal with $N_{u}$ users in the system is expressed as

$$
\begin{aligned}
r(t) & =\sum_{u=1}^{N_{u}} \sum_{l=0}^{L-1} \alpha_{u, l} s_{u}\left(t-l T_{p}\right)+\eta(t) \\
& =\sum_{l=0}^{L-1} \alpha_{1, l} s_{1}\left(t-l T_{p}\right)+\sum_{u=2}^{N_{u}} \sum_{l=0}^{L-1} \alpha_{u, l} s_{u}\left(t-l T_{p}\right)+\eta(t)(3
\end{aligned}
$$

where $\eta(t)$ is the additive white Gaussian noise process with a two-sided power spectral density (PSD) of $N_{0} / 2$ and $\alpha_{u, l}$ represents the channel coefficient of the $l^{\text {th }}$ path experienced by the $u^{t h}$ user's signal. The second term on the right-hand side of Eq. (3) is the multiple access interference (MAI) from
$N_{u}-1$ interfering users. The optimal receiver for the singleuser PPM system is a correlation receiver with a template waveform [7]

$$
\Delta_{u}(t)=S_{u, 0}(t)-S_{u, 1}(t) .
$$

Although this receiver is not optimum when MAI is present, it is adopted in this paper because of its simplicity.

\section{ERror PERformance ANALYSis}

We focus on the detection of the first bit of the desired user (user 1). Without loss of generality, we assume that the transmitted bit is a 1 , i.e., $s_{1}(t)=\sqrt{\mathcal{E}_{s}} S_{1,0}(t)$. With the large guard interval between adjacent symbols as mentioned in Section II-A, there is no ISI. We also assume synchronous reception of all users' signals ${ }^{1}$. The receiver, which includes a RAKE to combine (maximal ratio) the energies contained in resolvable muhtipath components, is assumed to have perfect knowledge of the desired user's channel coefficients that are combined by the RAKE.

For simplicity of notation, index for the desired user $(u=1)$ will be omitted is some variables whenever not to cause a confusion. Let the autocorrelation function of $S_{u, 0}(t)$ be

$$
\gamma_{u}(v) \triangleq \int_{-\infty}^{\infty} S_{u, 0}(t) S_{u, 0}(t-v) d t
$$

The signal, inter-path interference (self-noise), MAI, and additive noise components for the $k^{\text {th }}$ finger of the desired user at the input of the combiner are given as

$$
\begin{aligned}
Y_{s, k} & =\alpha_{1, k}^{2} \sqrt{\mathcal{E}_{s}} \int_{0}^{T_{r}} S_{u, 0}\left(t-k T_{p}\right) \Delta_{1}^{(k)}(t) d t \\
& =\alpha_{1, k}^{2} \sqrt{\mathcal{E}_{s}}\left[1-\gamma_{1}(\tau)\right] \\
Y_{s n, k} & =\sqrt{\mathcal{E}_{s}} \alpha_{1, k} \int_{0}^{T_{r}} \sum_{\substack{l=0 \\
l \neq k}}^{L-1} \alpha_{1, l} S_{1,0}\left(t-l T_{p}\right) \Delta_{1}^{(k)}(t) d t \\
Y_{m a i, k} & =\sqrt{\mathcal{E}_{s}} \alpha_{1, k} \int_{0}^{T_{r}} \sum_{u=2}^{N_{u}} \sum_{l=0}^{L-1} \alpha_{u, l} s_{n}\left(t-l T_{p}\right) \Delta_{1}^{(k)}(t) d t \\
Y_{\eta, k} & =\alpha_{1, k} \int_{0}^{T_{r}} \eta(t) \Delta_{1}^{(k)}(t) d t
\end{aligned}
$$

where $\Delta_{1}^{(k)}(t)=\Delta_{1}\left(t-k T_{p}\right)$ is the template waveform corresponding to the $k^{\text {th }}$ finger of the desired user. Conditioned on a set of channel coefficients of the desired user $\left\{\alpha_{1, k}\right\}$, the signal component, $Y_{s, k}$, is a constant. However, the self-noise, MAI, and additive noise are still treated as random variables (RVs). The inter-path interference has $(L-1)$ terms. Since $S_{u, 0}(t)$ consists of $N_{c}$ pulses (pulse width $T_{p}<T_{c}$ ) that are $T_{c}$ apart, the autocorrelation function $\gamma_{u}(v)$ is non-zero only for certain values of the argument $v$. Non-zero values of $\gamma_{u}(v)$ are centered around the local minima's and maxima's of $\gamma_{u}(v)$ that are $T_{c}$ apart. Inter-path interference is caused by multipath components, which are spaced at integer multiples

\footnotetext{
${ }^{1}$ Unlike in mobile communications, this assumption is reasonable for shortrange indoor communications for which propagation delays are negligible and users typically have a slow mobility.
} 
of $T_{c}$ from the $k^{t h}$ path that arrives before and after the $k^{t h}$ path.

The error performance becomes mathematically tractable if the number of paths combined by the RAKE is less than the ratio $\epsilon=T_{c} / T_{p}$. This ensures that interference is caused only by the $\left(N_{c}-1\right)$ paths arriving at integer multiples of $T_{c}$ after the $k^{\text {th }}$ path. Under this condition, the inter-path interference term, $Y_{s n, k}$, can be simplified as

$$
Y_{s n, k}=\sqrt{\mathcal{E}_{S}} \alpha_{1, k} \boldsymbol{\alpha}_{\mathbf{1}, k}{ }^{\prime} \gamma_{\mathbf{1}}
$$

where $[\cdot]^{\prime}$ denotes transpose and

$$
\begin{aligned}
& \boldsymbol{\alpha}_{\mathbf{1 , k}}=\left[\begin{array}{llll}
\alpha_{1, k+\epsilon}, & \alpha_{1, k+2 \epsilon}, & \ldots, & \alpha_{1, k+\left(N_{c}-1\right) \epsilon}
\end{array}\right]^{\prime} \\
& \gamma_{\mathbf{1}}=\left(\begin{array}{c}
\gamma_{1}\left(T_{c}\right)-\gamma_{1}\left(T_{c}-\tau\right) \\
\gamma_{1}\left(2 T_{c}\right)-\gamma_{1}\left(2 T_{c}-\tau\right) \\
\vdots \\
\gamma_{1}\left(\left[N_{c}-1\right] T_{c}\right)-\gamma_{1}\left(\left[N_{c}-1\right] T_{c}-\tau\right)
\end{array}\right)
\end{aligned}
$$

are $\left(N_{c}-1\right) \times 1$ vectors.

The user codes of interfering users are assumed to be independent of each other. With synchronous reception, MAI can be modeled in a way similar to the modeling of inter-path interference. The additive noise component is still a zero-mean Gaussian RV whose variance depends on $\alpha_{1, k}$. The $k^{t h}$ path to the combiner is expressed as $Y_{k}=Y_{s, k}+Y_{s n, k}+Y_{m a i, k}+Y_{\eta, k}$ and is a random variable. Variables $Y_{s n, k}, Y_{m a i, k}$ and $Y_{\eta, k}$ are zero-mean independent RVs, i.e., $E\left\{Y_{s n, k}\right\}=E\left\{Y_{m a i, k}\right\}=$ $E\left\{Y_{\eta, k}\right\}=0$ and $E\left\{Y_{s n, k} Y_{\eta, k}\right\}=0, E\left\{Y_{s n, k} Y_{m a i, k}\right\}=$ $0, E\left\{Y_{m a i, k} Y_{\eta, k}\right\}=0$, where $E\{\cdot\}$ denotes the statistical expectation. The mean and variance of $Y_{k}$ can be determined as

$$
\begin{aligned}
E\left\{Y_{k}\right\} & =Y_{s, k}=\alpha_{1, k}^{2} \sqrt{\mathcal{E}_{s}}\left[1-\gamma_{1}(\tau)\right] \\
\operatorname{Var}\left\{Y_{k}\right\} & =\operatorname{Var}\left\{Y_{s n, k}\right\}+\operatorname{Var}\left\{Y_{m a i, k}\right\}+\operatorname{Var}\left\{Y_{\eta, k}\right\} .
\end{aligned}
$$

Variance of the self-noise term is found by squaring $Y_{s n, k}$ and taking its expected value. Variable $Y_{s n, k}^{2}$ is a function of $\alpha_{1, i} \alpha_{1, j}$. Since the multipath components are assumed to be independent, $E\left\{\alpha_{1, i} \alpha_{1, j}\right\}=0$ for $i \neq j$. Hence, the variance of the self-noise term simplifies to

$$
\operatorname{Var}\left(Y_{s n, k}\right)=\mathcal{E}_{s} \alpha_{1, k}^{2} E\left\{\boldsymbol{\alpha}_{\mathbf{1}, \boldsymbol{k}}^{(2)^{\prime}}\right\} \boldsymbol{\Gamma}_{\mathbf{1}}
$$

where

$$
\begin{aligned}
& \boldsymbol{\alpha}_{\mathbf{1 , k}}^{(\mathbf{2})}=\left[\begin{array}{llll}
\alpha_{1, k+\epsilon}^{2}, & \alpha_{1, k+2 \epsilon}^{2}, & \ldots, & \alpha_{1, k+\left(N_{c}-1\right) \epsilon}^{2}
\end{array}\right]^{\prime} \\
& \boldsymbol{\Gamma}_{\mathbf{1}}=\left(\begin{array}{c}
\left\{\gamma_{1}\left(T_{c}\right)-\gamma_{1}\left(T_{c}-\tau\right)\right\}^{2} \\
\left\{\gamma_{1}\left(2 T_{c}\right)-\gamma_{1}\left(2 T_{c}-\tau\right)\right\}^{2} \\
\vdots \\
\left\{\gamma_{1}\left(\left[N_{c}-1\right] T_{c}\right)-\gamma_{1}\left(\left[N_{c}-1\right] T_{c}-\tau\right)\right\}^{2}
\end{array}\right)
\end{aligned}
$$

are $\left(N_{c}-1\right) \times 1$ vectors. It should be mentioned that in Eqs. (8) and (10) the autocorrelation values do not depend on $k$, the index of the path combined by the receiver. Following a similar procedure, variance of MAI can also be obtained except that the cross correlation vector must be averaged over user codes as well as transmitted symbols of interfering users. The variance of the MAI is of the form

$$
\operatorname{Var}\left(Y_{m a i, k}\right)=\mathcal{E}_{s} \sum_{u=2}^{N_{u}} \alpha_{1, k}^{2} E\left\{\boldsymbol{\alpha}_{\boldsymbol{u}, \boldsymbol{k}}^{(\mathbf{2})}{ }^{\prime} \boldsymbol{\Gamma}_{\boldsymbol{u}}\right\}
$$

where $N_{c} \times 1$ vectors $\boldsymbol{\alpha}_{\boldsymbol{u}, \boldsymbol{k}}^{(2)}$ and $\boldsymbol{\Gamma}_{\boldsymbol{u}}$ are given as

$$
\begin{gathered}
\boldsymbol{\alpha}_{\boldsymbol{u}, \boldsymbol{k}}^{(\mathbf{2})}=\left[\begin{array}{ccc}
\alpha_{u, k}^{2}, & \alpha_{u, k+\epsilon}^{2}, \quad \ldots, & \alpha_{u, k+\left(N_{c}-1\right) \epsilon}^{2}
\end{array}\right]^{\prime} \\
\boldsymbol{\Gamma}_{\boldsymbol{u}}=\left(\begin{array}{c}
\left\{\gamma_{u}(0)-\gamma_{u}(\tau)\right\}^{2} \\
\left\{\gamma_{u}\left(T_{c}\right)-\gamma_{u}\left(T_{c}-\tau\right)\right\}^{2} \\
\vdots \\
\left\{\gamma_{u}\left(\left[N_{c}-1\right] T_{c}\right)-\gamma_{u}\left(\left[N_{c}-1\right] T_{c}-\tau\right)\right\}^{2}
\end{array}\right)
\end{gathered}
$$

$\Gamma_{u}$ represents the cross correlation vector of the interfering users' signal with the desired user's signal. The variance of the additive noise term is given by,

$$
\operatorname{Var}\left(Y_{\eta, k}\right)=\alpha_{1, k}^{2} N_{0}\left[1-\gamma_{1}(\tau)\right]
$$

Assuming $K$ out of $L(K<\epsilon)$ resolvable paths are combined by the RAKE receiver, the output of the combiner is $Z=\sum_{k=0}^{K-1} Y_{k}=\sum_{k=0}^{K-1} Y_{s, k}+\sum_{k=0}^{K-1} Y_{s n, k}+$ $\sum_{k=0}^{K-1} Y_{m a i, k}+\sum_{k=0}^{\bar{K}-1} Y_{\eta, k}$. As mentioned earlier, under the assumption that $\left\{\alpha_{1,0}, \alpha_{1,1}, \ldots, \alpha_{1, K-1}\right\}$ is known at the receiver, the first term of $Z$, which is the signal component, is a constant. The second and third terms of $Z$, which are, respectively, the self-noise and MAI components from all the paths, is approximated as a Gaussian RV. The fourth term which is the additive noise is a zero-mean gaussian RV. Different paths to the combiner can be easily shown to be independent of each other because noise and interference in different paths are independent of each other. Hence $Z$ is a Gaussian RV with a mean and variance equal to the sum of the mean's and variance's of all the paths. Thus, mean and variance of $Z$ is obtained as

$$
\begin{aligned}
\mu_{Z}= & \sqrt{\mathcal{E}_{s}}\left[1-\gamma_{1}(\tau)\right] \sum_{k=0}^{K-1} \alpha_{1, k}{ }^{2} \\
\sigma_{Z}{ }^{2}= & \left\{N_{0}\left[1-\gamma_{1}(\tau)\right]+\mathcal{E}_{s} E\left\{\boldsymbol{\Lambda}_{\mathbf{1}}{ }^{\prime}\right\} \boldsymbol{\Gamma}_{\mathbf{1}}\right. \\
& \left.+\sum_{u=1}^{N_{u}} \mathcal{E}_{s} E\left\{\boldsymbol{\Lambda}_{\boldsymbol{u}} \boldsymbol{\Gamma}_{\boldsymbol{u}}\right\}\right\} \sum_{k=0}^{K-1} \alpha_{1, k}^{2}
\end{aligned}
$$

where $\left(N_{c}-1\right) \times 1$ vector $\boldsymbol{\Lambda}_{\mathbf{1}}$ is expressed as

$$
\boldsymbol{\Lambda}_{\mathbf{1}}=\left(\begin{array}{c}
\sum_{k=0}^{K-1} \alpha_{1, k+\epsilon}^{2} \\
\sum_{k=0}^{K-1} \alpha_{1, k+2 \epsilon}^{2} \\
\vdots \\
\sum_{k=0}^{K-1} \alpha_{1, k+\left(N_{c}-1\right) \epsilon}^{2}
\end{array}\right) .
$$

The $N_{c} \times 1$ vector $\boldsymbol{\Lambda}_{\boldsymbol{u}}$ is formed by stacking $\sum_{k=0}^{K-1} \alpha_{u, k}^{2}$ on top of $\Lambda_{1}$ with index 1 replaced by index $u$.

Now the conditional probability of error can be obtained as $Q\left(\mu_{Z} / \sqrt{\sigma_{Z}^{2}}\right)$ where $Q(\cdot)$ is the $Q$-function defined as $Q(x)=\int_{x}^{\infty} \frac{1}{\sqrt{2 \pi}} e^{-x^{2} / 2} d x$.

The conditional probability of error is

$$
\begin{aligned}
& P_{e \mid\left\{\alpha_{1, k}\right\}}= \\
& Q\left(\sqrt{\frac{E_{S}[1-\gamma(\tau)]^{2} \varphi}{N_{0}[1-\gamma(\tau)]+\mathcal{E}_{s} E\{\boldsymbol{\Lambda}\} \boldsymbol{\Gamma}+\sum_{n=1}^{N_{u}} \mathcal{E}_{s} E\left\{\boldsymbol{\Lambda}^{n} \boldsymbol{\Gamma}_{\boldsymbol{c}}\right\}}}\right)
\end{aligned}
$$


where $\varphi=\sum_{k=0}^{K-1} \alpha_{1, k}^{2}$. To obtain the average probability of error, the conditional probability of error in Eq. (15) can be averaged over the probability density function (PDF) of the random variable $\varphi$. Each of the $\left\{\alpha_{1, k}\right\}$ in $\varphi$ is a lognormal random variable and so is $\left\{\alpha_{1, k}^{2}\right\}$. Hence $\varphi$ is a sum of lognormal random variables. A closed-form expression for the PDF of a sum of lognormal random variables is not available. Many methods can be applied to accurately approximate the sum of independent lognormal RVs as another lognormal RV [10] [11]. One such method is the Wilkinson's method, which has been proved to be very accurate in certain regions of the argument where most of the practical problems fall.

Let the lognormal RV $\alpha_{1, k}=e^{y_{k}}$ where $y_{k} \sim$ $N\left(\mu_{y_{k}}, \sigma_{y_{k}}{ }^{2}\right)$ is normally distributed. According to Wilkinson's method, $\varphi$ can be written as $\varphi=e^{R}$, where $R \sim$ $N\left(\mu_{R}, \sigma_{R}^{2}\right)$ is also normally distributed. Mean and standard deviation of $R$ are obtained as $\mu_{R}=\ln \left(\xi_{L 1}{ }^{2} / \sqrt{\xi_{L 2}}\right)$ and $\sigma_{R}=\sqrt{\ln \left(\xi_{L 1}{ }^{2} / \xi_{L 2}\right)}$. The two quantities $\xi_{L 1}$ and $\xi_{L 2}$ are related to $y_{k}$ as,

$$
\begin{aligned}
\xi_{L 1} & =\sum_{k=0}^{K-1} e^{\left(2 \mu_{y_{k}}+2 \sigma_{y_{k}}^{2}\right)} \\
\xi_{L 2} & =\sum_{k=0}^{K-1} e^{2\left(2 \mu_{y_{k}}+4 \sigma_{y_{k}}{ }^{2}\right)} \\
& +2 \sum_{k=0}^{K-2} \sum_{n=k+1}^{K-1} e^{2\left(\mu_{y_{k}}+\mu_{y_{n}}+\sigma_{y_{k}}{ }^{2}+\sigma_{y_{n}}{ }^{2}\right)}
\end{aligned}
$$

Hence, the PDF of the approximated lognormal random variable is written as

$$
f(\varphi)=\frac{1}{\varphi \sqrt{2 \pi \sigma_{R}^{2}}} \exp \left[-\frac{\left\{\ln (\varphi)-\mu_{R}\right\}^{2}}{2 \sigma_{R}^{2}}\right]
$$

The average BER can be calculated by averaging the conditional BER $P_{e \mid\left\{\alpha_{1, k}\right\}}$ over the PDF $f(\varphi)$ as $P_{e}=$ $\int_{0}^{\infty} P_{c /\{\alpha\}} f(\varphi) d \varphi$.

\section{Numerical RESUlts AND Discussions}

For the numerical examples, the channel is assumed to have an exponentially decaying multipath intensity profile (MIP) and the receiver is assumed to have perfect knowledge of the channel coefficients of the desired user. The number of resolvable paths $(L)$ and the power decay factor $(\rho)$ are obtained using the channel root-mean-square (RMS) delay spread as described in [6]. For a typical indoor environment, the channel standard deviation is in the range of $3-5 d B$. All paths whose power are within $15 d B$ of that of the first path are considered. For an RMS delay spread of $20 \mathrm{~ns}$, the total resolvable paths was obtained to be $L=179$ with a corresponding power decay factor $\rho=0.019$. All users (desired and interfering users) have the same received bit energy. The sum of power of all paths that are combined by the RAKE is normalized to unity. Thus, for a given signalto-noise ratio (SNR), the variance of AWGN is obtained as $N_{0} / 2=10^{-S N R / 10}$. Pulse width of $0.5 n s$, user code of length 32, data rate of $2.5 \mathrm{Mbps}$ (which includes a guard interval of 100ns), channel RMS delay spread of $20 \mathrm{~ns}$ are adopted in all examples unless explicitly specified otherwise.

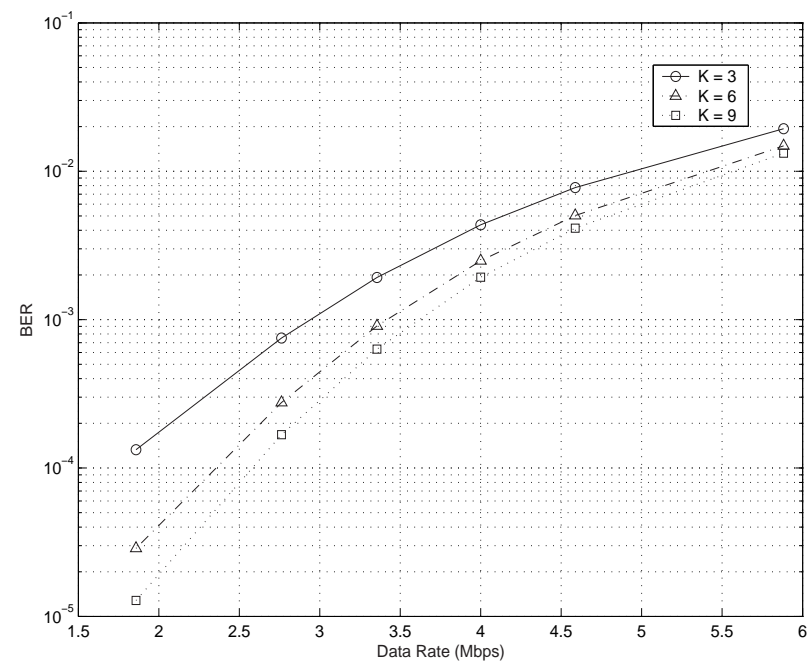

Fig. 1. Performance of the optimally-spaced scheme with diffent $K$.

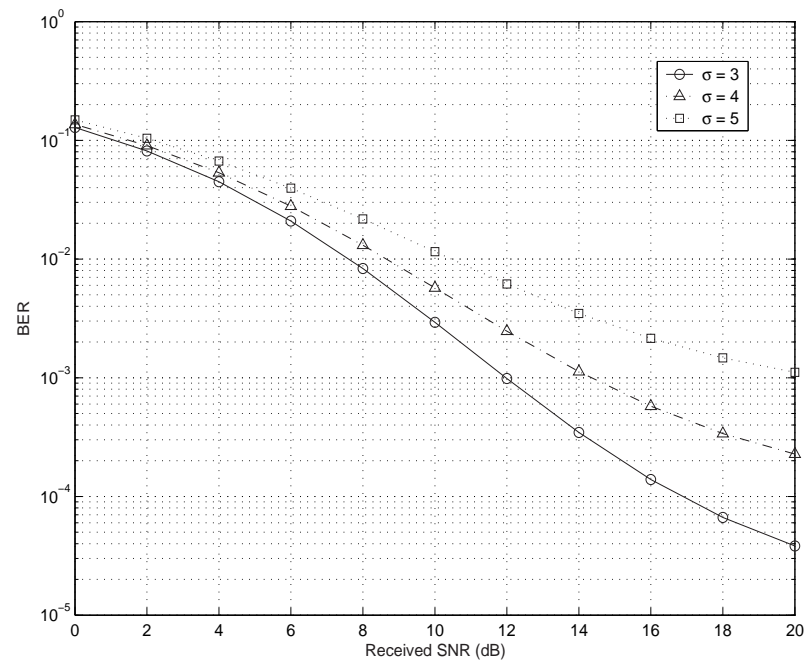

Fig. 2. Performance of the optimally-spaced scheme for different $\sigma$.

Fig. 1 shows the single-user performance of the optimallyspaced scheme at an SNR of $15 d B$. BER curves are given for different number of paths $(K)$ that are combined by the RAKE. It is found that performance improvement from $K=6$ to $K=9$ is small compared to the improvement from $K=3$ to $K=6$ at low data rates.

Fig. 2 illustrates the performance of a single-user system with the optimally-spaced scheme for different standard deviations $(\sigma)$ of the channel with $K=5$. The scheme exhibits an error floor at high SNR values. A smaller $\sigma$ results in a lower error floor and it seems that the impacts of $\sigma$ on the error performance is significant for the set of system parameters chosen.

To assess the performance improvement of the optimallyspaced scheme over that of the orthogonal signaling schemes, we plotted, in Fig. 3, the SNR versus BER curves for a singleuser system with different $K$. It is observed that with the same set of parameters, the optimally-spaced scheme has a much lower error floor than the orthogonal scheme.

Fig. 4 shows the performance of a single-user system with the optimally-spaced signaling scheme. BER curves shown are 


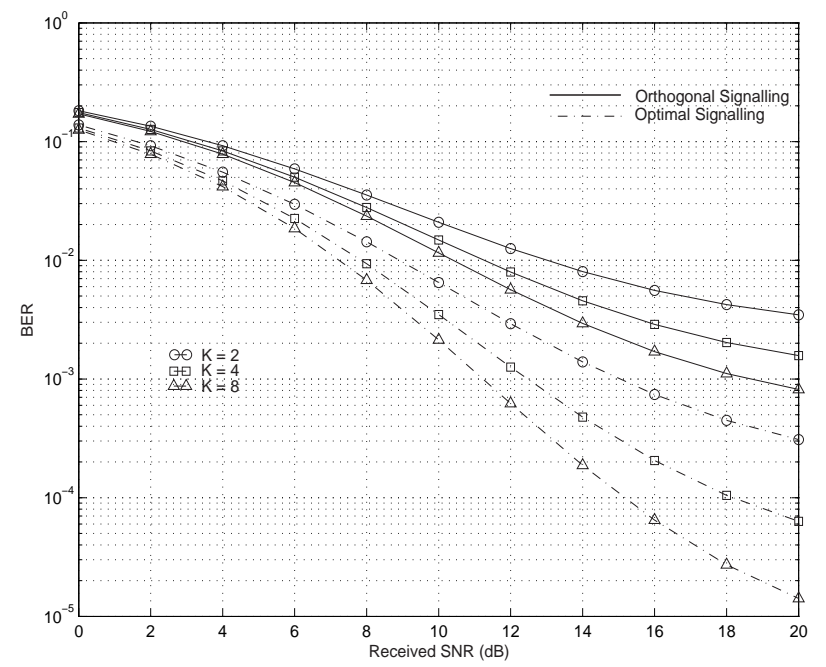

Fig. 3. Performance comparison of the optimally-spaced and the orthogonal signaling schemes.

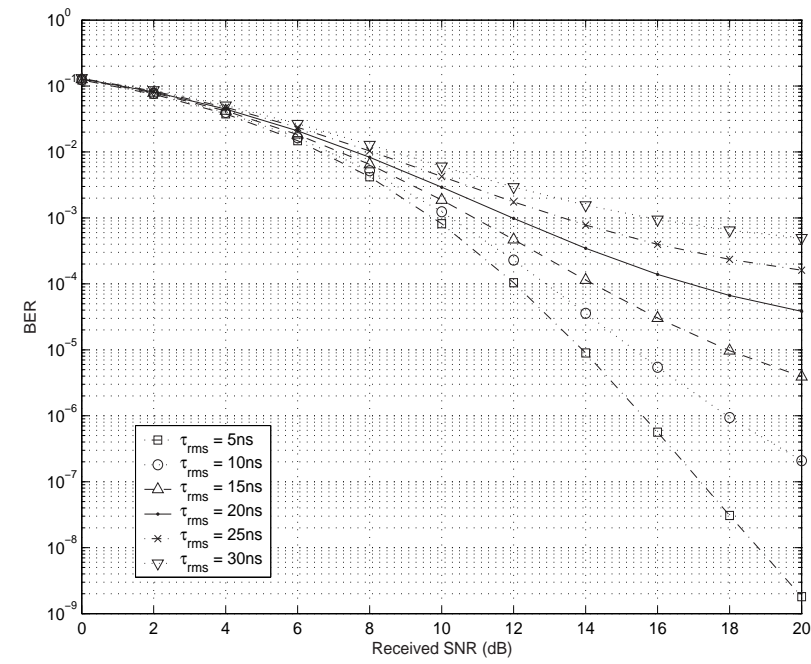

Fig. 4. The impact of channel RMS delay spread $\left(\tau_{r m s}\right)$.

for $K=5$ with different values of the channel delay spread $\tau_{r m s}$. For high $\tau_{r m s}$ values (e.g., greater than 20ns), an error floor appears at a $20 d B$ SNR. The scheme works well for small $\tau_{r m s}$.

The effects of MAI on the error performance of the system performance is shown in Fig. 5. BER curves as a function of the number of interfering users $\left(N_{u}-1\right)$ are given for an SNR of $20 d B$ and $K=3,6$, and 9 . When the number of interfering users are large (e.g., greater than 10), BER deteriorates significantly and increasing the number of paths combined does not improve the performance.

\section{CONCLUSIONS}

Through the analytical BER expression derived for a PPM multi-user UWB system with DS spreading for multiple access, we have demonstrated that the performance of such a system is limited by inter-path and multiple-access interferences at high SNR values. With reasonably large SNR values, the optimally-spaced PPM scheme has a much lower error floor than the orthogonal signaling scheme. Channel

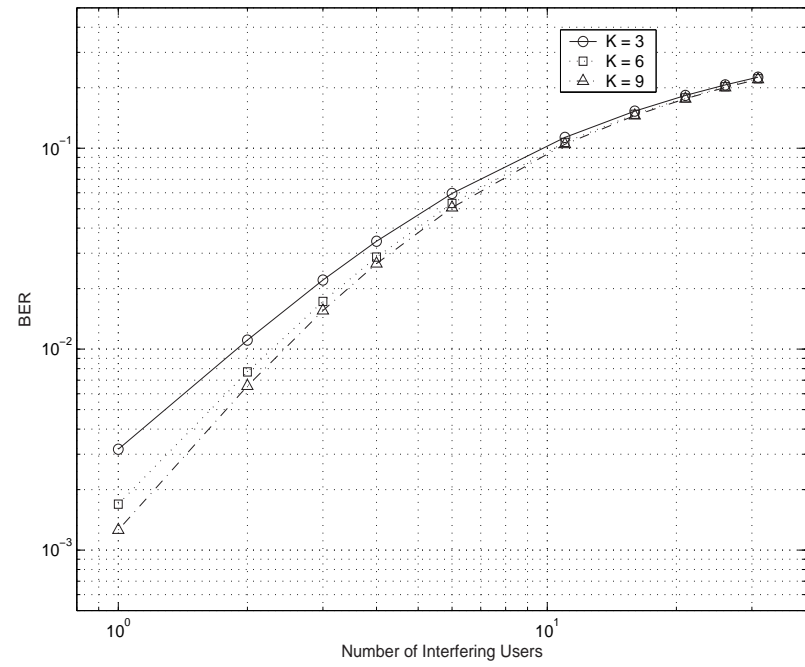

Fig. 5. The effect of multiple access interference.

delay spread values have been found to significantly affect the BER performance. Due to direct sequence spreading, the time interval between adjacent chips of the same symbol decreases. This causes inter-path interference, which limits the performance of the system.

\section{REFERENCES}

[1] M. Win and R. Scholtz, "Ultra-wide bandwidth timing-hopping spread spectrum impulse radio for wireless multiple-access communications," IEEE Trans. on Communications, vol. 48, pp. 36-38, Apr. 2000.

[2] J. R. Foerster, "The performance of a direct-sequence spread spectrum ultra-wideband system in the presence of multipath, narrowband interference and multiuser interference," Proc. of the 2002 IEEE Conference on Ultra Wideband Systems and Technologies, May 2002, pp. 87-91.

[3] J. Foerster and Q. Li, "UWB channel modeling contribution from Intel," IEEE P802.15-02/279-SG3a.

[4] H. Hasmemi, "Inpulse response modeling of indoor radio propagation channels," IEEE Journal on Selected Areas in Communications, vol. 11, pp. 967-978, Sept. 1993.

[5] H. Hashemi, "The indoor radio propagation channel," Proc. of the IEEE, vol. 81, pp. 943-967, July 1993.

[6] J. R. Foerster, "The effects of multipath interference on the performance of UWB systems in an indoor wireless channel," Proc. of IEEE VTC 2001-Spring, vol. 2, 2001, pp. 1176-1180.

[7] R. Scholtz, "Multiple access with time-hopping impulse modulation," Proc. of MILCOM'93, vol. 2, 1993, pp. 447-450.

[8] J. G. Proakis, Digital Communications (ch. 14). New York, NY: McGrawHill, 3rd ed., 1995.

[9] L. Rusch, C. Prettie, D. Chueng, Q. Li and M. Ho, "Characterization of UWB propagation from 2 to $8 \mathrm{GHz}$ in a residential environment," submitted to IEEE Journal on Selected Areas in Communications, Dec. 2001.

[10] N. C. Beaulieu, A. A. Abu-Dayya, and P. J. Mclane, "Estimating the distribution of a sum of independent lognormal random variable," IEEE Trans. on Communications, vol. 43, pp. 2869-2873, Dec. 1995.

[11] S. B. Slimane, "Bounds on the Distribution of a Sum of Independent Lognormal Random Variables," IEEE Trans. on Communications, vol. 49, pp. 975-978, Jun. 2001. 